\title{
Perfil Epidemiológico da Síndrome de Down no Estado da Bahia
}

\author{
Juliana Gonçalves Cunha \\ Acadêmica do $5^{\circ}$ ano do Curso de Fisioterapia da \\ Escola Bahiana de Medicina e Saúde Pública \\ (juliana_gcunha@msn.com) \\ Acadêmica do $5^{\circ}$ ano do Curso de Fisioterapia da \\ Naiane de Oliveira Costa \\ Escola Bahiana de Medicina e Saúde Pública \\ (nfisio@yahoo.com.br) \\ Bióloga, Doutora em Saúde Pública, Professora \\ Marlene Silva \\ Adjunta do Curso de Fisioterapia da Escola Bahiana \\ de Medicina e Saúde Pública \\ (msilva@bahiana.edu.br) \\ Tipo de Pesquisa Artigo Original \\ Origem da Pesquisa \\ Trabalho de Conclusão de Curso de Fisioterapia da \\ Escola Bahiana de Medicina e Saúde Pública \\ Tipo de Análise do Manuscrito Triple Blind Peer Review \\ Recebido em Mai/11 Aprovado em Jun/11
}

\section{Resumo}

Introdução: A Síndrome de Down (SD) é uma desordem genética, caracterizada pela trissomia do cromossomo 21, cujo fator que indubitavelmente se associa com a SD é a idade materna avançada. Entretanto, características especiais da mãe ou das condições de nascimento são encontradas com mais freqüência entre tais crianças do que entre as crianças normais. 0 objetivo deste estudo é traçar o perfil epidemiológico de recém-nascidos com SD, no Estado da Bahia; verificar as relações entre as características maternas e de nascimento e a SD, além de identificar possíveis associações do Apgar e do peso ao nascer com a SD. Material e Método: 0 estudo é transversal, com uma série temporal de recém-nascidos (RN) no estado da Bahia, entre 2005 e 2007, registrados no Sistema de Informação de Nascidos Vivos (SINASC). Resultados: Entre os 671.650 nascidos vivos a maior incidência de SD foi observada em 2005. Fatores como idade avançada, escores baixos de Apgar no 1으 e no 50 minutos, baixo peso ao nascer e idade gestacional prematura apresentaram associações estatisticamente significantes as maiores incidências da Síndrome. Conclusão: mostrou-se que RN com SD apresentam características comuns evidenciando a necessidade do estímulo precoce. Apesar dos resultados, sugerem-se novos estudos com mesmos fatores, maior período, em diferentes populações.

Palavras-chave: Síndrome de Down; incidência; perfil epidemiológico; nascimentos na Bahia; Apgar 
Epidemiological Profile of Down syndrome in the State of Bahia

\begin{abstract}
Introduction: Down syndrome (DS) is a genetic disorder characterized by trisomy of chromosome 21 , which undoubtedly factor that is associated with the syndrome is advanced maternal age. However, the special characteristics of the mother or the conditions of birth are more frequently found among these children than among normal children. The objective of this study is to evaluate the epidemiological profile of infants with Down syndrome in the state of Bahia, to examine relationships between maternal characteristics and birth and SD and to identify possible associations of the Apgar score and birth weight with DS. Material and Methods: The study is transversal, with a series of newborns (NB) in the state of Bahia, between 2005 and 2007, recorded in the Information System on Live Births (SINASC). Results: Among 671,650 live births to higher incidence of SD was observed in 2005. Factors such as age, low Apgar scores at 1 and at 5 minutes, low birth weight and gestational age premature show statistically significant association of the highest incidences of the syndrome. Conclusion: it was shown that infants with Down syndrome have common features highlighting the need for early stimulation. Despite the results, we suggest further studies with these factors, longer, in different populations.
\end{abstract}

Keywords: Down Syndrome, incidence, epidemiology, births in Bahia, Apgar

\title{
Perfil epidemiológico del síndrome de Down en el Estado de Bahía
}

\section{Resumen}

Introducción: El síndrome de Down (SD) es un trastorno genético caracterizado por la trisomía del cromosoma 21, que, sin duda, factor que se asocia con el síndrome de Down es la edad materna avanzada. Sin embargo, las características especiales de la madre o las condiciones de luz son más frecuentes entre los niños que entre los niños normales. El objetivo de este estudio es evaluar el perfil epidemiológico de los niños con síndrome de Down en el estado de Bahía, para examinar las relaciones entre las características maternas y el nacimiento y la SD, y para identificar posibles asociaciones entre la puntuación de Apgar y el peso al nacer con síndrome de Down. Material y Métodos: El estudio es transversal, con una serie de recién nacidos (RN) en el estado de Bahia, entre 2005 y 2007, registrados en el Sistema de Información sobre Nacidos Vivos (SINASC). Resultados: De los 671.650 nacidos vivos a una mayor incidencia de SD se observó en 2005. Factores como la edad, las puntuaciones bajas de Apgar a 1 y en 5 minutos, bajo peso al nacer y edad gestacional prematura muestran una asociación estadísticamente significativa de los índices más altos del síndrome. Conclusión: Se ha demostrado que los bebés con síndrome de Down tienen características comunes destacan la necesidad de estimulación temprana. A pesar de los resultados, le sugerimos que otros estudios con estos factores, ya, en diferentes poblaciones.

Palabras clave: Síndrome de Down, la incidencia, epidemiología, los nacimientos en Bahía, Apgar

\section{Le profil épidémiologique du syndrome de Down dans l'état de Bahia}

\section{Résumé}

Introduction: Le syndrome de Down (SD) est un trouble génétique caractérisée par une trisomie du chromosome 21, qui a sans doute facteur qui est associé au syndrome est l'âge 
maternel avancé. Toutefois, les caractéristiques particulières de la mère ou les conditions de naissance sont plus fréquemment retrouvés chez ces enfants que chez les enfants normaux. L'objectif de cette étude est d'évaluer le profil épidémiologique de nourrissons atteints du syndrome de Down dans l'État de Bahia, pour examiner les relations entre les caractéristiques de la mère et la naissance et SD et d'identifier les associations possibles du score d'Apgar et les poids à la naissance avec la DS. Matériel et Méthodes: L'étude est transversale, avec une série de nouveaux-nés (N.-B.) dans l'Etat de Bahia, entre 2005 et 2007, enregistrées dans le Système d'information sur les naissances vivantes (SINASC). Résultats: Parmi les 671650 naissances vivantes à forte incidence de SD a été observée en 2005. Des facteurs tels que l'âge, les scores d'Apgar bas à 1 et à 5 minutes, faible poids de naissance et l'âge gestationnel montrent prématurée association statistiquement significative de la plus haute incidence de ce syndrome. Conclusion: il a été démontré que les nourrissons atteints du syndrome de Down ont des caractéristiques communes soulignant la nécessité de la stimulation précoce. Malgré les résultats, nous suggérons des études complémentaires à ces facteurs, plus, dans différentes populations.

Mots-clés: Syndrome de Down, l'incidence, l'épidémiologie, les naissances, à Bahia, d'Apgar

\section{Introdução}

A Síndrome de Down (SD) é uma desordem genética, caracterizada pela trissomia do cromossomo 21, gerando 47 cromossomos ao invés de 46.1 A SD causa características faciais ${ }^{2}$ e físicas, que se manifestam em graus variáveis.3 Exemplo dessas alterações é o pescoço largo e curto, mãos largas e curtas, nariz pequeno, hipotonia muscular, frouxidão ligamentar e instabilidade da articulação atlantoaxial.3 Além disso, é a causa mais comum de retardo mental.1,4 Esta síndrome tem uma ocorrência relativamente elevada. Nos Estados Unidos nascem aproximadamente 5 mil bebês com SD anualmente, com uma incidência de um a cada 7 mil nascidos vivos. ${ }^{1}$ No Brasil, essa incidência sobe para um em cada 600/800 nascidos vivos, sendo encontrados no censo de 2000, 3.000 portadores no país. ${ }^{5}$ Estudo sobre perfil epidemiológico das malformações congênitas no município de Vitória-ES, mostrou que a SD contribuiu com 7,4\% das anomalias estudadas e, 6 comparativamente ao Sudeste, o Nordeste brasileiro apresentou maior risco para a SD.7

A incidência de SD aumenta com a idade materna ${ }^{8}$, a qual se designa avançada, a partir dos 35 anos. ${ }^{7}$ Comprovadamente, este é o único fator de risco para a sua ocorrência, ${ }^{9}$ embora não seja descartada nascimentos com SD entre mães jovens, 10 bem como o aumento da incidência da SD, com a ausência de diagnóstico precoce no pré-natal e exposição à radiação ionizante. ${ }^{10}$ Além disso, como conseqüência da idade materna avançada, está a ocorrência do Apgar baixo,11,12 o qual é utilizado para mensurar a vitalidade do recém-nascido. ${ }^{13}$ Contudo, não foi encontrado estudos que avaliassem o perfil de Apgar em crianças com SD. Ademais, as informações sobre fatores que levam a SD ainda são escassas. A revisão bibliográfica no tema constata um déficit de dados epidemiológicos específicos, e principalmente, uma deficiência em estudos que buscam avaliar os efeitos do da Síndrome e seus possíveis comprometimentos no neonato.

Tudo isso, motivou o presente estudo, que traça o perfil epidemiológico de recémnascidos com Síndrome de Down, no estado da Bahia, em um breve período, verificando as relações entre as características maternas e da criança com a SD, além de identificar se existe associação entre os escores de Apgar e peso ao nascer com tal desordem. 


\section{Material \& Método}

Trata-se de estudo transversal e descritivo de séries temporais, que analisa o perfil epidemiológico de recém-nascidos (RN) com Síndrome de Down no estado da Bahia, nos anos 2005 a 2007 . Incluiu-se o total de RN vivos no período e excluíram-se aqueles com anomalia que não fosse a SD e os que não apresentavam a CID da anomalia congênita.

Os dados foram coletados do banco do Sistema de Informação de Nascidos Vivos (SINASC), que contém registros dos nascimentos, desde 1979.1 Para a descompactação e leitura dos bancos utilizou-se o programa TAB para Windows. Após este procedimento os bancos de cada ano foram transferidos para o programa SPSS2 pacote estatístico para as ciências sociais (SPSS) versão 17.0. A partir disso, foram selecionadas as variáveis referentes aos RN, como o Apgar no primeiro e no quinto minuto (Quadro 1), o peso ao nascer, a idade gestacional, o sexo, a raça/cor; bem como as referentes a parturiente, como idade, escolaridade, tipo de gravidez e número de consultas pré-natal.

A variável de interesse principal foi definida como com SD, reunindo seus códigos Classificação Internacional de Doenças (CID 10), agrupadas em Q90.0: trissomia 21, não disjunção meiótica; Q90.1: trissomia 21, mosaico (nã̃o disjunção mitótica); Q90.2: trissomia 21, translocação; Q90.9: Síndrome de Down não especificada. Os demais foram agrupados como sem anomalia.

Anterior à análise, limpou-se o banco, imputando dados ignorados e perdidos com base na medida de maior frequência, por ano estudado, independente de ter ou não a Síndrome. Na variável anos de estudo, os dados perdidos foram registrados em "até 7 anos", nos nascimentos de 2005 e 2006 e registrado como "8 anos e mais", em 2007. Quanto à idade materna, imputou-se na faixa etária entre 16 a 25 anos, pois obteve maior frequência de nascimentos em todos os anos. Já para o índice de Apgar no primeiro e no quinto minuto, foram imputados na categoria "de 8 a 10". A idade gestacional (IG) perdida foi substituída por "37 a 41 semanas". Independente do grupo, o "peso ao nascer" foi imputado em "mais que $2.500 \mathrm{~g}$ " e o "tipo de gravidez" perdido foi substituído por "única" em todo o período estudado. 0 "número de consultas" sem registro passou a ser "até 6" nos três anos de estudo, os campos sem sexo passaram a ser masculino e "raça/cor" os dados foram imputados na categoria "parda".

$\mathrm{Na}$ análise estatística, foram calculadas as medidas descritivas de tendência central e as de dispersão (variáveis contínuas) e medidas de freqüência absoluta e relativa (variáveis categóricas) de acordo os anos e grupos estudados. Após isso, categorizaram-se as variáveis contínuas e recategorizaram algumas categóricas para imprimir melhor poder ao estudo nas inferências. Estimou-se a Incidência de RN com SD nas categorias das variáveis, em cada ano e as possíveis diferenças foram identificadas por meio do teste do Qui-Quadrado de Mantel Hanszel, com base em valor de $p$, considerando um alfa menor que 0,05.

\section{Resultados}

0 estudo incluiu 671.650 nascidos vivos, no estado da Bahia, no período de 2005 a 2007, dos quais foram excluídos 83.746 RN apresentado diagnóstico de anomalia congênita que não era a Síndrome de Down ou por ausência do registro da CID da anomalia congênita. Em 2005, o número de RN foi discretamente maior (231.065), a incidência de SD variou de acordo com o sexo e os anos observados, sendo maior em 2005 (1,81/10.000 nascidos vivos) e entre as meninas, em todo o período (Figura 1).

\footnotetext{
10 acesso foi através do site www.datasus.gov.br, selecionando-se e gravando os bancos de dados do estado da Bahia, dos anos de 2005, 2006 e 2007.

${ }^{2}$ SPSS $=$ Statistical Package for the Social Sciences.
} 
Entre as características maternas, viu-se que mães destas crianças eram mais velhas variando a distribuição entre $35,9 \%$ a 58,1\% (36 e mais anos) entre os anos; embora na faixa de até 25 anos de idade, tenha encontrado de 26,2\% a 41,1\% das crianças com SD. A distribuição da escolaridade mostrou-se irregular entre os grupos em todos os anos estudados. Em geral, os percentuais de crianças em que as mães fizeram maior número de consultas de pré-natal foram discretamente mais elevados no grupo sem anomalias do que no grupo de RN com SD (Tabela 1).

As características dos RN (Tabela 2) distribuíram-se diferentemente entre os grupos. Comparativamente, em todos os anos os percentuais de crianças com SD foram maiores nos escores de Apgar (10 min. e 5ำ min.) mais baixos, e menores nos outros; por exemplo, no Apgar 10 minuto - escore de 0-3 observou-se 6,5\% de crianças com SD frente a 1,2\% de crianças sem anomalia ou no escore 8-10 (58,1\% crianças com SD/ 80,4\% crianças sem anomalia), assemelhando-se ao que ocorreu no Apgar no 5ำ minuto em que a distribuição e diferenças dos percentuais persistiram, embora tenham sido menores. Enquanto 20,5\% a $29,0 \%$ dos RN com SD apresentaram baixo peso, apenas $6,6 \%$ das crianças sem anomalia estava nesta faixa; notaram-se poucas diferenças na distribuição dos RN(s) de acordo com a IG e raça/cor, mas chamou atenção o aumento no percentual $(14,3 \%)$ de RN com até 36 semanas no grupo com SD do que no sem anomalias $(5,2, \%)$, no ano de 2005 , bem como o maior percentual de RN da raça/cor preta $(7,1 \%)$ no grupo com SD quando comparado ao sem anomalia $(2,8 \%)$ no mesmo ano. 0 percentual de meninas foi maior no grupo com SD, confrontando o grupo sem anomalia.

Analisando-se as Incidências de SD de acordo as características incluídas neste estudo (Tabela 3), se identificou que a idade materna esteve associada ao nascimento de crianças com SD em todos os anos. Verificaram-se maiores incidências de SD (IC de SD) entre os RN(s) com mães a partir de 36 anos variando de 10,75/10.000 RN a 14,21/10.000 RN entre os anos. Estas diferenças foram estatisticamente significantes $(p=0,00)$.

A Incidência de SD também diferiu em níveis estatisticamente significantes, de acordo com os escores do Apgar no $1^{\mathbf{0}}$ minuto (exceto 2007), no 5ํㅡㄴ minuto, do peso ao nascer e da IG (exceto $2007-p=0,08$ ), em quase todos os anos estudados. Enquanto a Incidência de SD foi de 9 RN em 10.000 RN com escore de 0-3, no Apgar 10 minuto, foi observado apenas 1 RN com SD em 10.000 RN no escore de 8-10. Na avaliação do Apgar no 50 minuto, apenas no ano de 2005 foi observado RN com SD com escore de Apgar 0-3 (9,41/10.000 RN), nos demais anos a incidência de SD mostrou-se maior em escores de Apgar mais elevados (de 4 a 7). Quanto menor o peso ao nascer, maior foi a Incidência da SD, com destaque a maior IC de SD observada entre os RN(s) com muito baixo peso (IC=10,06/10.000 RN), em 2005.

Além disso, verificou-se também que a Incidência de SD era maior em RN prematuros. Especificamente nos anos 2005 e 2006 ocorreram diferenças nas IC de SD de acordo com a IG $(\mathrm{p}=0,02)$, observando-se maior incidência de SD entre os RNs pré-termo (até 36 semanas) que variou de 3,84 em 2007 a 5,85 em 2005 (Tabela 4)

\section{Discussão}

Este estudo mostra uma incidência de SD entre os nascidos vivos baixa na Bahia, entre 2005 e 2007, apesar de variar discretamente entre os anos e ser maior entre as meninas. Além disso, confirmou-se que idade materna maior que 35 anos é fator de risco para o nascimento de criança com SD. Crianças com SD apresentaram Apgar baixo no primeiro e no quinto minuto, baixo peso ao nascer e prematuridade, sugerindo que a SD afeta as características da gestação e do nascimento. Fatores como escolaridade, número de consultas pré-natal, raça/cor e sexo possivelmente não guardam relação com a Síndrome.

Diferentemente da Bahia, a maioria dos estudos mostram que a incidência de SD é encontrada em valores mais elevados. Verificando-se freqüentes relatos de que a estimativa global da SD é de cerca de 1 em 600 nascidos vivos, ${ }^{14}$ o que representa 16 a cada 10.000 RN, 
embora sejam informações desatualizadas. Contudo, recentemente, baseados dados do SINASC, são mostrados valores um pouco maiores do que a observada na Bahia. Por exemplo, incluindo nascimentos de 2001 a 2004 em Vitória (ES), foram encontrados 5 casos de SD,6 que representou uma incidência de aproximadamente de 2,8/10.000 RN e no Vale do ParaíbaSP, os 14 casos identificados, representaram uma Incidência de SD de $3,4 / 10.000$, entre os nascimentos de 2002 e 2003. ${ }^{15}$

Vale ressaltar que a escassez de estudos nacionais sobre anomalias congênitas dificulta ampliar esta discussão, especialmente quanto aos fatores associados à SD. Tem-se abordados globalmente a malformação, e não se identificam a dimensão dos tipos de Anomalias e de seus fatores determinantes. Por exemplo, no Rio Grande do Sul foram identificados 45 casos de malformações congênitas entre 1985 a 1995 (4,34 por 1.000 nascidos vivos)16, em Vitória-ES foram 68 casos (IC 3,9/1.000 RN) entre 2001 a 20046 e no Vale do Paraíba (SP), em um menor período (2002 e 2003) foram relatados 317 casos destas alterações globais $(7,6$ por 1.000), valores acima dos encontrados nos demais estudos. ${ }^{16}$

Esta pesquisa confirma que a idade materna avançada é um fator de risco para a ocorrência da SD, corroborando com estudo de 220 casos de SD no Serviço de Genética/Laboratório de Genética Humana e Citogenética do Instituto de Biologia da Universidade Federal da Bahia,7 em que foi encontrada alta correlação ( $r=0,95)$ entre estas variáveis, em 2003.17 Mulheres em idade avançada ${ }^{6}$ apresentam diminuição da função ovariana e redução do número de oócitos, levando a uma baixa fertilidade. ${ }^{12}$ Este fenômeno se inicia após os 30 anos intensificando-se após os 40.18

Os baixos escores de Apgar tanto no primeiro quanto no quinto minuto, mais comum entre as crianças com SD em relação as crianças sem anomalias mostram que o desempenho ao nascimento é influenciado diretamente pela SD. Em Vitória-ES ${ }^{6}$ as crianças apresentaram similar padrão de distribuição dos níveis de escores, embora os RN com malformações tenham apresentado estimativas de menores escores de Apgar em maior magnitude. Um índice de Apgar no quinto minuto, de 3 ou menos, implica um risco significativamente maior de ter algum tipo de deficiência, incluindo atraso no desenvolvimento, déficit na atenção, praxia fina e transtornos relacionados a hiperatividade, apontando para uma necessidade de recursos adicionais na escola, 19 bem como direcionamento precoce ao atendimento fisioterapeutico. Convém informar que a maioria das crianças apresentam índices de Apgar satisfatórios, independente de ter SD ou outras malformações congênitas.6,15 Entretanto, existe uma correlação entre a idade da mãe e escores de Apgar. Segundo autores que avaliaram os efeitos da idade na gravidez, mulheres mais velhas apresentam maior risco de um bebê com baixos índices de Apgar, mesmo sem malformações congênitas. ${ }^{12}$

Quanto ao peso, foi mais comum crianças com SD nas categorias de baixo de peso ao nascer do que entre os demais RN, o que está de acordo estudo realizado na cidade de São Paulo através da análise de prontuários, obtidos em consultas de pacientes com SD, no qual mostrou que em geral, os RN com SD apresentavam $500 \mathrm{~g}$ a menos no peso comparados aos RN sem SD.17 Os achados do presente estudo indicam que a presença da SD leva a um menor peso da criança, o que possivelmente é conseqüência de um nascimento prematuro e/ou do atraso no crescimento durante a vida intrauterina, já que também se observa na literatura uma maior incidência de SD no grupo de muito baixo peso e de baixo peso. ${ }^{20} \mathrm{~A}$ associação entre malformações congênitas e baixo peso também foi encontrada no Vale do Paraíba Paulista. ${ }^{15}$

Embora o peso ao nascer seja uma característica comprometida entre as crianças com qualquer anomalia congênita, a maioria com SD na Bahia, se concentrou na categoria "maior que $2.500 \mathrm{~g}$ ", semelhantemente ao ocorrido entre as malformações congênitas, cuja média foi de 3.006g.6 Em especial, as mães mais velhas necessitam de um acompanhamento adequado na gravidez, ${ }^{18}$ promovendo a redução da incidência de baixo peso ao nascer. Como neste estudo não foi observada relação da SD com o número inadequado de consultas de pré-natal, é possível sugerir que a ocorrência de baixo peso nas crianças analisadas esteja, de fato, relacionada à SD e não as características ou comportamento maternos. 
A importante diferença encontrada na ocorrência de SD de acordo com as semanas de gestação em 2005 e 2006 permite afirmar que a SD acarreta nascimento prematuro da criança. Tal achado também foi encontrado em relação às malformações congênitas que se associaram com a menor duração da gestação no estudo de Vitória-ES em que a Incidência de malformação entre os RN com 22 a 36 semanas foi o dobro $(6,47 / 1.000)$ da encontrada em RN com 37 semanas e mais (IC=3,6/1.000). ${ }^{6}$ Todavia, esta associação não foi observada nas crianças com malformação nascidas no Vale do Paraíba Paulista. ${ }^{15}$ Embora não esclareçam adequadamente os mecanismos, estudos mostram que a malformação genética compromete o perfeito desenvolvimento intrautero provocando prematuridade. ${ }^{15}$

Ressalta-se que a indisponibilidade de dados acerca de outras patologias possivelmente apresentadas pelas crianças com Síndrome de Down limitou o entendimento das associações entre o baixo peso, os índices de Apgar e a prematuridade com a SD, visto que é possível que estes efeitos tenham sido decorrentes de outras enfermidades da mãe, concomitantes à SD.

\section{Conclusão}

Este estudo apresenta resultados consistentes das características comuns aos RN com Síndrome de Down, os quais poderão auxiliar em um melhor preparo na assistência ao parto e ao pós-parto, através do estímulo à reabilitação precoce visto que SD, prematuridade e apresentar muito baixo peso podem levar ao atraso no desenvolvimento neuromotor da criança. 21 Apesar dos importantes resultados aqui encontrados, sugere-se novos estudos focalizando estas mesmas variáveis em diferentes populações e em maior intervalo de anos, bem como constante capacitações de profissionais envolvidos no preenchimento de informações nas declarações de nascidos vivos e no SINASC, a fim de evitar a subnotificação dos casos, uma vez que podem ter sido perdidos casos de SD por estarem sem CID. Além disso, recomenda-se a inclusão de outras informações no SINASC, como história de doenças maternas na gestação, pois poderia mostrar outros fatores associados à ocorrência da SD, visto que há relatos na literatura de maior probabilidade de malformações em filhos de mães com asma brônquica, por exemplo. ${ }^{15}$

\section{Referências}

1.TECKLIN, I S. Fisioterapia Pediátrica. (3rd ed), São Paulo-SP: Artmed, 2002, 250p.

2. JAOUAD, I C. et al. "Cytogenetic and Epidemiological Profiles of Down Syndrome in a Moroccan Population: a report of 852 cases", Morocco: Singapore Medice Journal, 5(2): 133-36. 2010.

3. BERTHOLD, T M; et al. "Síndrome de Down: Aspectos Gerais e Odontológicos", Salvador-BA: Revista de Ciências Médicas e Biológicas, 3(2): 252-60. 2004.

4. GHOSH S; FEINGOLD E \& DEY, S. "Etiology of Down Syndrome: Evidence for Consistent Association Among Altered Meiotic Recombination, Nondisjunction and Maternal Age Across Populations", India: Americam Journal of Medical Genetics, 149(7): 1415-20. 2009.

5. SILVA, M, \& KLEINHANS, S. "Processos Cognitivos, Plasticidade Cerebral e Síndrome de Down", Marília-SP: Revista Brasileira de Educação Especial, 12(1): 123-38. 2006.

6. MACIEL, E L N; et al. "Perfil Epidemiológico das Malformações Congênitas no Município de VitóriaES", Rio de Janeiro-RJ: Caderno de Saúde Coletiva, 14(3): 507-18. 2006.

7. GUSMÃO, F, TAVARES, E, \& MOREIRA, L. "Idade Materna e Síndrome de Down no Nordeste do Brasil", Rio de Janeiro-RJ: Caderno de Saúde Pública, 19(4): 973-78. 2003.

8. RIBEIRO, L M A; et al. “Avaliação dos Fatores Associados a Infecções Recorrentes e/ou Graves em Pacientes com Síndrome de Down”, Rio de Janeiro-RJ: Jornal de Pediatria, 79(2): 141-48. 2003.

9. OTEIZA, L M E F; et al. "Tendencias del Sindrome de Down en Cuba. Su Relación con Edad Materna y Tasa de Fecundidad", Cuba: Revista Cubana Pediatria, 70(3): 141-47. 1998.

10. SILVA, N \& DESSEN, M. "Síndrome de Down: etiologia, caracterização e impacto na família", BrasíliaDF: Interação em Psicologia, 6(2): 167-76. 2002.

11. SANTOS, G H N; et al. "Impacto da Idade Materna sobre os Resultados Perinatais e Via de Parto", São Luís-MA: Revista Brasileira de Ginecologia e Obstetrícia, 31(7): 326-34. 2009. 
12. CECATTI, J G; et al. "O Impacto da Idade Materna Avançada sobre os Resultados da Gravidez", Campinas-SP: Revista Brasileira de Ginecologia e Obstetrícia, 20(7): 1998.

13. KILSZTAJN, S; et al. "Vitalidade do Recém-Nascido por Tipo de Parto no Estado de São Paulo", Rio de Janeiro-RJ: Caderno de Saúde Pública, 23(8): 1886-92. 2007.

14. HERNANDEZ, D. \& FISHER, E. "Down Syndrome Genetics: unravelling a multifactorial disorder", Human Molecular Genetics, 5: 1411-16. 1996.

15. PINTO, C. \& NASCimenTO, L. “Estudo de Prevalência de Defeitos Congênitos no Vale do Paraíba Paulista", Guaratinguetá-SP: Revista Paulista de Pediatria, 25(3): 233-39. 2007.

16. LEITE, J. \& SCHÜLER-FACCINI, L. "Defeitos Congênitos em uma Região de Mineração de Carvão", São Paulo-SP: Revista de Saúde Pública, 35(2): 136-41. 2001.

17. PRADO, M B. et al. “Acompanhamento Nutricional de Pacientes com Síndrome de Down Atendidos em um Consultório Pediátrico", São Paulo-SP: O Mundo da Saúde, 33(3): 335-34. 2009.

18. LIMA, L C. “Idade Materna e Mortalidade Infantil: efeitos nulos, biológicos ou socioeconômicos?” Rio de Janeiro-RJ: Revista Brasileira de Estudo das Populações, 27(1): 211-26. 2010.

19. MOSTER, D; LIE, R. \& MARKESTAD, T. "Joint Association of Apgar Scores and Early Neonatal Symptoms with Minor Disabilities at School Age", Bergen: Archive Disease Child Fetal Neonatal, 86(1): 16-21. 2002.

20. RIBEIRO, A M; et al. "Fatores de Risco para Mortalidade Neonatal em Crianças com Baixo Peso ao Nascer", Recife-PE: Revista de Saúde Pública, 43(2): 246-55. 2009.

21. FLABIANO, F C; BÜHLER, K E C B; \& LIMONGI, S C O. "Desenvolvimento cognitivo e de linguagem expressiva em um par de gêmeos dizigóticos: influência da síndrome de Down e da prematuridade associada ao muito baixo peso", São Paulo-SP: Revista da Sociedade Brasileira de Fonoaudiologia, 14(2): 267-74. 2009

\section{Apêndice}

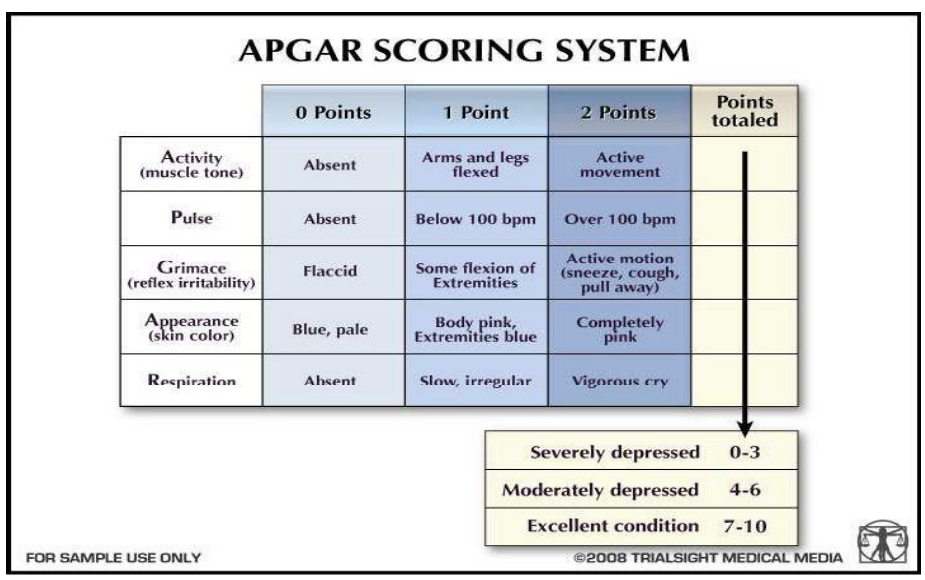

Quadro 1

Significado do escore de Apgar 
Figura

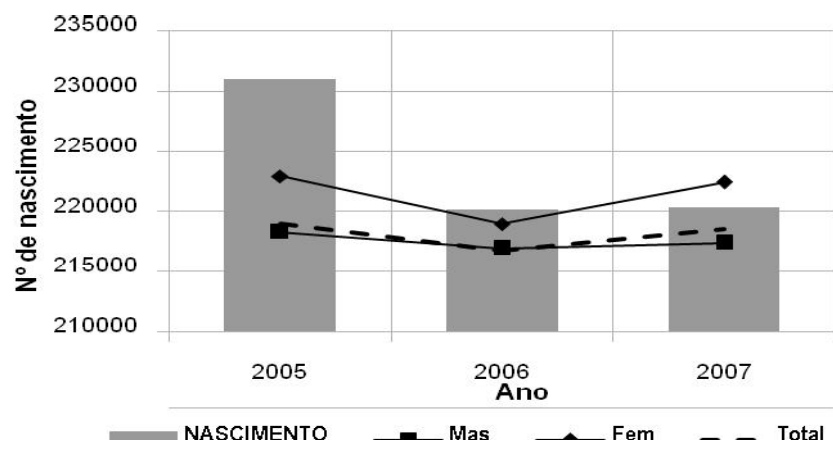

Número de nascidos vivos e incidência de SD por sexo e anos de nascimento na Bahia, 2005-2007

Tabela 1

Características das mães de nascidos vivos com Síndrome de Down e sem anomalias congênitas, na Bahia, no período de 2005 a 2007 ( $\mathrm{N}=671.650)$

\begin{tabular}{|c|c|c|c|c|c|c|c|c|c|c|c|c|}
\hline \multirow{4}{*}{$\begin{array}{l}\text { Características } \\
\text { Maternas }\end{array}$} & \multicolumn{12}{|c|}{ Ano Calendário de Nascimento } \\
\hline & \multicolumn{4}{|c|}{2005} & \multicolumn{4}{|c|}{2006} & \multicolumn{4}{|c|}{2007} \\
\hline & \multicolumn{2}{|c|}{$\begin{array}{c}\text { Síndrome } \\
\text { de Down }\end{array}$} & \multicolumn{2}{|c|}{ Sem Anomalia } & \multicolumn{2}{|c|}{$\begin{array}{l}\text { Síndrome } \\
\text { de Down }\end{array}$} & \multicolumn{2}{|c|}{ Sem Anomalia } & \multicolumn{2}{|c|}{$\begin{array}{l}\text { Síndrome } \\
\text { de Down }\end{array}$} & \multicolumn{2}{|c|}{ Sem Anomalia } \\
\hline & $\mathrm{N}=42$ & \begin{tabular}{|l|l|}
$0,01 \%$ \\
\end{tabular} & $\mathrm{~N}=231.023$ & $99,98 \%$ & $\mathrm{~N}=30$ & $0,01 \%$ & $\mathrm{~N}=220.157$ & $99,98 \%$ & $\mathrm{~N}=38$ & $0,01 \%$ & $\mathrm{~N}=220.360$ & $99,98 \%$ \\
\hline \multicolumn{13}{|c|}{ Idade materna (anos) } \\
\hline Até $15 \quad 0$ & 0 & 0 & 065 & 3,1 & 0 & 0 & 6.348 & 3,3 & 1 & 2,6 & 6.560 & 3,3 \\
\hline 16 a 25 & 26 & 119 & 9.232 & 60,3 & 9 & 29,0 & 113.910 & 58,8 & 15 & 38,5 & 112.068 & 57,1 \\
\hline 26 a 35 & 33 & 3,3 & .935 & 30,3 & 4 & 12,9 & 60.831 & 31,4 & 9 & 23,1 & 64.663 & 32,9 \\
\hline 36 e mais & 40 & 0,5 & 2.529 & 6,3 & 18 & 58,1 & 12.647 & 6,5 & 14 & 35,9 & 13.006 & 6,6 \\
\hline \multicolumn{13}{|c|}{ Escolaridade (anos de estudo) } \\
\hline Nenhum & 3 & 7,1 & 8.323 & 4,3 & 1 & 3,2 & 6.233 & 3,2 & 0 & 0 & 5.546 & 2,8 \\
\hline Até 7 & 23 & 54,8 & 108.586 & 55,9 & 13 & 41,9 & 104.658 & 54,0 & 23 & 59,0 & 93.679 & 47,7 \\
\hline 8 e mais & 16 & 38,1 & 77.284 & 39,8 & 17 & 45,8 & 82.845 & 42,8 & 16 & 41,0 & 97.072 & 49,5 \\
\hline \multicolumn{13}{|c|}{ Consultas pré-natal } \\
\hline Até 6 & 30 & 76,2 & 134.863 & 68,6 & 21 & 67,7 & 127.380 & 65,7 & 25 & 64,1 & 127.822 & 65,1 \\
\hline 7 e mais & 11 & 26,8 & 61.679 & 31,4 & 10 & 32,3 & 663.556 & 34,3 & 14 & 35,9 & 68.475 & 34,9 \\
\hline \multicolumn{13}{|c|}{ Tipo de Gravidez } \\
\hline \begin{tabular}{l|l} 
Única \\
\end{tabular} & 42 & 100 & 194.127 & 98,2 & 31 & 100 & 190.238 & 98,2 & 38 & 97,4 & 192.578 & 98,1 \\
\hline Dupla & 0 & 0 & 3.441 & 1,7 & 0 & 0 & 3.394 & 1,8 & 0 & 0 & 3.643 & 1,9 \\
\hline Tripla & 0 & 0 & 193 & 0,1 & 0 & 0 & 103 & 0,1 & 1 & 2,6 & 75 & 0,0 \\
\hline
\end{tabular}


Tabela 2

Características dos recém-nascidos com Síndrome de Down e sem anomalias congênitas, na Bahia, entre 2005 e 2007 ( $N=671.650)$

\begin{tabular}{|c|c|c|c|c|c|c|c|c|c|c|c|c|}
\hline \multirow{4}{*}{$\begin{array}{l}\text { Características } \\
\text { do neonato }\end{array}$} & \multicolumn{12}{|c|}{ Ano calendário de nascimento } \\
\hline & \multicolumn{4}{|c|}{2005} & \multicolumn{4}{|c|}{2006} & \multicolumn{4}{|c|}{2007} \\
\hline & \multicolumn{2}{|c|}{$\begin{array}{c}\text { Síndrome de } \\
\text { Down }\end{array}$} & \multicolumn{2}{|c|}{ Sem anomalia } & \multicolumn{2}{|c|}{$\begin{array}{c}\text { Síndrome de } \\
\text { Down }\end{array}$} & \multicolumn{2}{|c|}{ Sem anomalia } & \multicolumn{2}{|c|}{$\begin{array}{c}\text { Síndrome de } \\
\text { Down }\end{array}$} & \multicolumn{2}{|c|}{ Sem anomalia } \\
\hline & $\mathrm{N}=42$ & $0,01 \%$ & $\mathrm{~N}=231.023$ & $99,98 \%$ & $\mathrm{~N}=\mathbf{3 0}$ & $0,01 \%$ & $\mathrm{~N}=220.157$ & $99,98 \%$ & $\mathrm{~N}=38$ & $0,01 \%$ & $\mathrm{~N}=220.360$ & $99,98 \%$ \\
\hline \multicolumn{13}{|l|}{ Apgar (escore) } \\
\hline \multicolumn{13}{|l|}{$1 \%$ minuto } \\
\hline 0 a 3 & 2 & 4,8 & 2.688 & 1,4 & 2 & 6,5 & 2.322 & 1,2 & 2 & 5,1 & 2.818 & 1,3 \\
\hline 4 a 7 & 15 & 35,7 & 38.050 & 19,2 & 11 & 35,5 & 35.715 & 18,4 & 8 & 20,5 & 34.487 & 17,6 \\
\hline 8 a 10 & 25 & 59,5 & 157.023 & 79,4 & 18 & 58,1 & 155.699 & 80,4 & 29 & 74,4 & 159.292 & 81,1 \\
\hline \multicolumn{13}{|l|}{ 50 minuto } \\
\hline 0 a 3 & 1 & 2,4 & 1.061 & 0,5 & 0 & 0 & 758 & 0,4 & 0 & 0 & 836 & 0,4 \\
\hline 4 a 7 & 6 & 14,3 & 7.037 & 3,6 & 5 & 16,1 & 7.507 & 3,9 & 5 & 12,8 & 6.749 & 3,4 \\
\hline 8 a 10 & 35 & 83,3 & 189.663 & 95,9 & 26 & 83,9 & 185.471 & 97,7 & 34 & 87,2 & 188.712 & 96,1 \\
\hline \multicolumn{13}{|l|}{ Peso ao nascer } \\
\hline Muito baixo & 2 & 4,8 & 1.986 & 1,0 & 0 & 0 & 2.310 & 1,2 & 1 & 2,6 & 2.355 & 1,2 \\
\hline Baixo & 12 & 28,6 & 13.052 & 6,6 & 9 & 29,0 & 12.632 & 6,5 & 8 & 20,5 & 12.955 & 6,6 \\
\hline Normal & 28 & 66,7 & 182.723 & 92,4 & 22 & 71,0 & 178.794 & 92,3 & 30 & 76,9 & 180.987 & 92,2 \\
\hline \multicolumn{13}{|c|}{ Semanas de Gestação } \\
\hline Até 36 & 6 & 14,3 & 10.244 & 5,2 & 5 & 16,1 & 10.536 & 5,4 & 0 & 0 & 5.546 & 2,8 \\
\hline 37 a 41 & 36 & 85,7 & 183.866 & 93,3 & 26 & 83,9 & 180.226 & 93,0 & 23 & 59,0 & 93.679 & 47,7 \\
\hline 42 e mais & 0 & 0 & 2.873 & 1,5 & 0 & 0 & 2.974 & 1,5 & 16 & 41,0 & 97.072 & 49,5 \\
\hline \multicolumn{13}{|l|}{ Raça/Cor } \\
\hline Branca & 4 & 9,5 & 24.776 & 12,5 & 3 & 10,0 & 24.520 & 12,7 & 4 & 10,5 & 21.289 & 10,8 \\
\hline Preta & 3 & 7,1 & 5.487 & 2,8 & 0 & 0 & 4.316 & 2,2 & 1 & 2,6 & 3.915 & 2,0 \\
\hline Amarela & 1 & 2,4 & 780 & 0,4 & 0 & 0 & 612 & 0.3 & 0 & 0 & 410 & 0,2 \\
\hline Parda & 34 & 81,0 & 166.111 & 84,0 & 27 & 90,0 & 163.873 & 84,6 & 33 & 86,8 & 170.264 & 86,7 \\
\hline Indígena & 0 & 0 & 607 & 0,3 & 0 & 0 & 415 & 0,2 & 0 & 0 & 419 & 0,2 \\
\hline \multicolumn{13}{|l|}{ Sexo } \\
\hline Masculino & 17 & 40,5 & 101.542 & 51,3 & 14 & 46,7 & 99.738 & 51,5 & 15 & 39,5 & 100.295 & 51,1 \\
\hline Feminino & 25 & 59,5 & 96.219 & 48,7 & 16 & 53,3 & 94.008 & 48,5 & 23 & 60,5 & 96.002 & 48,9 \\
\hline
\end{tabular}

Tabela 3

Incidência de Síndrome de Down de acordo com os fatores maternos, na Bahia, entre 2005 e 2007 
Tabela 4

Incidência de Síndrome de Down de acordo características do neonato, na Bahia, entre 2005 e 2007

\begin{tabular}{|c|c|c|c|c|c|c|}
\hline \multirow{3}{*}{ Características do neonato } & \multicolumn{6}{|c|}{ Ano calendário de nascimento } \\
\hline & \multicolumn{2}{|l|}{2005} & \multicolumn{2}{|c|}{2006} & \multicolumn{2}{|c|}{2007} \\
\hline & Incidência* & p-valor & Incidência* & p-valor & Incidência* & p-valor \\
\hline \multicolumn{7}{|l|}{ Apgar (escore) } \\
\hline 10 minuto & & 0,00 & & 0,00 & & 0,08 \\
\hline 0 a 3 & 7,43 & & 8,60 & & 7,93 & \\
\hline 4 a 7 & 3,94 & & 3,07 & & 2,02 & \\
\hline 8 a 10 & 1,59 & & 1,09 & & 1,82 & \\
\hline 5o minuto & & 0,00 & & 0,00 & & 0,00 \\
\hline 0 a 3 & 9,41 & & 0 & & 0 & \\
\hline 4 a 7 & 8,51 & & 6,65 & & 7,40 & \\
\hline 8 a 10 & 1,84 & & 1,34 & & 1,74 & \\
\hline Peso ao nascer & & 0,00 & & 0,00 & & 0,00 \\
\hline Muito baixo & 10,06 & & 0 & & 4,24 & \\
\hline Baixo & 9,18 & & 7,11 & & 6,17 & \\
\hline Normal & 1,53 & & 1,17 & & 1,60 & \\
\hline Semanas de Gestação & & 0,02 & & 0,02 & & 0,31 \\
\hline Até 36 & 5,85 & & 4,74 & & 3,84 & \\
\hline 37 a 41 & 1,95 & & 1,38 & & 1,85 & \\
\hline 42 e mais & 0 & & 0 & & 0 & \\
\hline Raça/Cor & & 0,10 & & 0,87 & & 0,99 \\
\hline Branca & 1,61 & & 1,22 & & 1,87 & \\
\hline Preta & 5,46 & & 0 & & 2,55 & \\
\hline Amarela & 12,80 & & 0 & & 0 & \\
\hline Parda & 2,04 & & 1,70 & & 1,99 & \\
\hline Indígena & 0 & & 0 & & 0 & \\
\hline
\end{tabular}

